Research paper

\title{
Lifetime reproductive output in a hermaphrodite cestode when reproducing alone or in pairs: a time cost of pairing
}

\author{
LUKAS SCHÄRER ${ }^{1,3 *}$ and CLAUS WEDEKIND ${ }^{1,2,4}$ \\ ${ }^{1}$ Abteilung Verhaltensökologie, Zoologisches Institut, Universität Bern, 3032 Hinterkappelen, \\ Switzerland; ' Department of Biology, University of Utah, Salt Lake City, Utah 84112-0840, USA; \\ Present address: ${ }^{3}$ Laboratoire d'Écologie, CC237, Université P\&M Curie, 7quai St. Bernard, 75252 \\ Paris Cedex 05, France; ${ }^{4}$ Institute of Cell, Animal and Population Biology, University of Edinburgh, \\ Kings Buildings, West Mains Road, Edinburgh EH9 3JT, Scotland, UK \\ (*author for correspondence, fax:+331442735 16; e-mail: lukas.scharer@snv.jussieu.fr)
}

Received 4 August 1999; accepted 22 March 2000

Co-ordinating editor: L.D. Hurst

\begin{abstract}
The cestode Schistocephalus solidus is a facultatively self-fertilising simultaneous hermaphrodite. Here we test for differences in the starting point, the rate, and the magnitude of egg production between individuals allowed to reproduce alone (only self-fertilisation possible) or in pairs (both self- and cross-fertilisation possible). Specifically, we want to distinguish between alternative processes responsible for the lower egg production in paired individuals observed in an earlier study (Wedekind et al., 1998). We designed an improved in vitro system, replacing the bird final host that allows us to measure, with high temporal resolution, the timing and magnitude of lifetime egg production of worms in these two social situations. We found that the experimental groups did not differ significantly in the starting point of egg production. However, the temporal pattern in egg production differed between them, in that paired individuals had a lower rate of egg production. This, however, did not lead to a significant reduction in lifetime egg production, as pairs compensated for the lower rate by producing eggs longer than single individuals. We argue that the lower rate of egg production may nevertheless lead to a time cost of pairing in the study species, and that this cost is likely to represent a cost of outcrossing due to sexual selection.
\end{abstract}

Key words: co-operation, cross-fertilisation, gamete trading, self-fertilisation, sex allocation, simultaneous hermaphroditism

\section{Introduction}

Much progress has been made in understanding how the reproductive biology of dioecious organisms has been shaped by sexual selection (Andersson and Iwasa, 1996; Cunningham and Birkhead, 1998). This is in sharp contrast to our understanding of this selective force in hermaphrodites, particularly simultaneous hermaphrodite animals (Charnov, 1979; Michiels, 1998; Greeff and Michiels, 1999a,b) where only few model systems exist. A large group of 
simultaneous hermaphrodites are the parasitic platyhelminths (i.e. cestodes, trematodes, monogeneans) many of which can reproduce by both self- and cross-fertilisation (review in Nollen, 1983). Low or unpredictable population densities and limited control over access to mating partners are likely to be selecting for both simultaneous hermaphroditism and the ability for selffertilisation in this group of animals (Tomlinson, 1966; Ghiselin, 1969; Jarne and Charlesworth, 1993). We may, however, also expect selection against selffertilisation, because self-fertilisation can lead to reduced fitness compared to cross-fertilisation in both animals and plants (e.g. Charlesworth and Charlesworth, 1987; Jarne and Delay, 1990; Schmitt and Ehrhardt, 1990).

Interestingly, several parasitic helminths employ both self- and cross-fertilisation when worms mate in groups (Nollen, 1975; Trouvé et al., 1996; Nollen, 1997), indicating that self-fertilisation can be a valid option in a situation where cross-fertilisation is possible. This may lead to sperm competition between own sperm and foreign sperm for the fertilisation of own eggs (Michiels, 1998). Sperm competition may, in turn, lead to an increase in male allocation in pairs over isolated (i.e. purely selfing) individuals (Charnov, 1982). Under the assumption of a trade-off between male and female investment, this may lead to a reduced female allocation, and may represent a cost of sexual selection (Lloyd, 1980; Lewis, 1987). Certain behavioural mechanisms may reduce the intensity of sperm competition, such as conditional, reciprocal gamete trading (Michiels, 1998). Reproductive strategies employed in facultatively selfing simultaneous hermaphrodites are therefore expected to depend on the presence or absence of a mating partner, and the costs and benefits associated with self- vs. crossfertilisation.

Wedekind et al. (1998) investigated reproductive parameters between individuals of the simultaneous hermaphrodite tapeworm Schistocephalus solidus (Müller, 1776). Worms were allowed to reproduce in an in vitro system replacing the intestine of the final host, while being kept isolated (singles, only self-fertilisation possible) or in groups of two (pairs, both self- and crossfertilisation possible). Pairs produced fewer eggs until 3 days after the start of the experiment. This difference was partly compensated by pairs producing larger eggs, but total produced egg mass (egg number times egg size) was also smaller in pairs. These differences suggest (a) that it is likely that different reproductive modes are employed in the two social situations, (b) that the different modes are associated with different reproductive strategies, and (c) that there may be cost of outcrossing as outlined above. Another line of evidence for a difference in the reproductive modes employed in the two situations comes from a recent histological study (L. Schärer, and C. Wedekind, unpubl. ms.). We found significant differences between isolated and paired worms for (a) sperm stored in the seminal vesicles (to be used in insemination), and (b) sperm stored in the seminal receptacles (received by insemination). 
The aim of the present study was to specifically address the origin of the difference in produced egg mass, and to relate it to differences in the timing and/or magnitude of egg production. This required designing an improved in vitro system that allowed to measure, with high temporal resolution, the dynamics of lifetime egg production of singles and pairs. Cumulative egg production can be described as a temporal process with a defined starting point, an initial increase in egg production rate, and a latter decrease in rate until an asymptote, reflecting total lifetime egg production, is reached. Temporal patterns between singles and pairs could differ in the ways outlined in Figure 1.

\section{Materials and methods}

\section{Study species}

The pseudophyllidean cestode $S$. solidus is a simultaneous hermaphrodite that reproduces in the intestine of fish eating birds. Eggs are passed out into the water with the faeces. If the free swimming first larval stage, the coracidium, is ingested by the first intermediate host, a cyclopoid copepod, the second larval stage, the procercoid, develops in the hemocoel of this host. Infectivity to the second intermediate host, the three-spined stickleback, Gasterosteus aculeatus, is reached within 1 to 2 weeks and infection occurs upon ingestion of the infected copepod by the fish. The third larval stage, the plerocercoid, grows in the peritoneum of the fish and reaches infectivity to the final host after 1 to 3 months. At this stage larvae are fully segmented and the genitalia are differentiated but immature (Smyth, 1946). Spermatogenesis only takes place in the final host and is triggered by the high body temperature of the final host (Smyth, 1952), ruling out the possibility of sperm transfer in the fish. After ingestion by the final host, the larvae mature and start to produce eggs within 2 days (Smyth, 1946). In vivo, reproduction takes place within 1 to 2 weeks, after which the worms die (McCaig and Hopkins, 1963; Tierney and Crompton, 1992). The short reproductive period facilitates measurement of lifetime egg production in this species.

In vitro system

The improved in vitro system (modified from Smyth, 1954 and Wedekind, 1997) consists of nylon mesh bags $(10 \times 80 \mathrm{~mm}, 200 \mu \mathrm{m}$ mesh size $)$ suspended into modified $50 \mathrm{ml}$ centrifuge tubes filled with culture medium (Wedekind, 1997). During the experiment the $\mathrm{pH}$ of the medium never dropped below 7 as assessed by the color of the added $\mathrm{pH}$ indicator. All material used in the experiment was thoroughly watered in deionized water for at least a week at $40{ }^{\circ} \mathrm{C}$ prior to the experiment. Tubes were mounted onto a horizontal shaker 


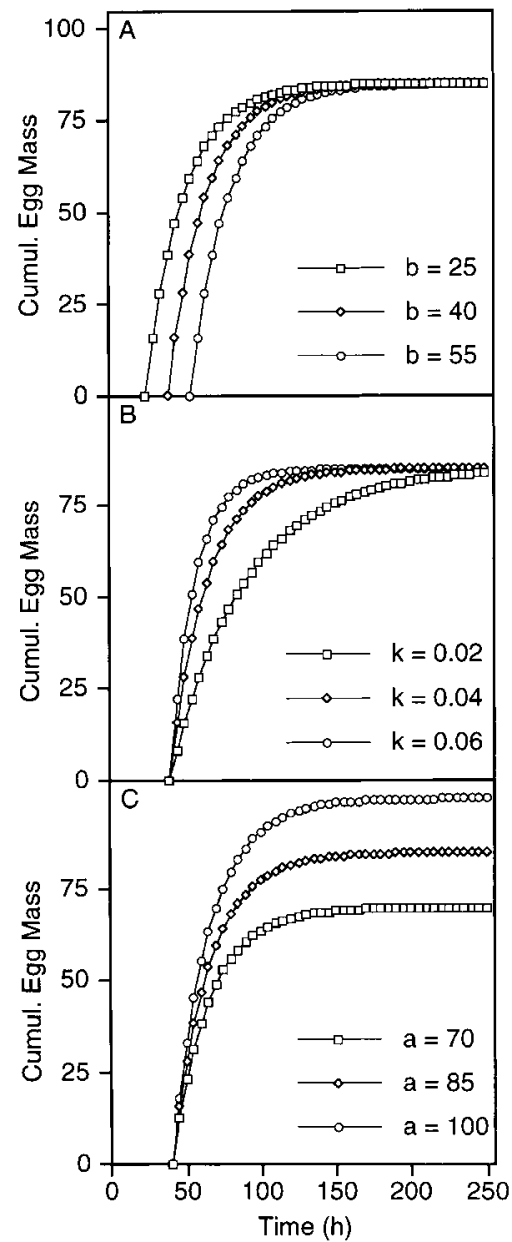

Figure 1. Computer generated von Bertalanffy growth curves, $y=a *(1-\exp (-k *(x-b)))$ are shown in order to visualise the properties of this growth function, and how they can be related to cumulative egg production in $S$. solidus. A: Processes affecting the starting point of egg reproduction. Effect of changing parameter $b$ while holding constant parameters $k$ and $a$. Higher values of $b$ in paired individuals would indicate delayed outcrossing and could arise from time spent for mate choice, mate assessment and the need for information transfer between mating partners. Higher values of $b$ in isolated individuals could indicate delayed self-fertilisation. B: Processes affecting the rate of egg production. Effect of changing parameter $k$ while holding constant $b$ and $a$. Lower values of $k$ in paired individuals indicate a lower rate of egg production and could be caused by mating behaviour in pairs requiring more time, for example due to gamete trading, due to a difference in the mechanism of sperm transfer, due to larger eggs taking longer to produce and/or due to an effect of crowding. C: Processes affecting the magnitude of egg production. Effect of changing parameter $a$ while holding parameters $b$ and $k$ constant. Lower values of $a$ in paired individuals indicate a reduced female allocation and could be due to an overall lower allocation to gametes caused by a higher energetic costs of copulation in paired individuals and/or due to an increased male allocation because of sperm competition. 
(80/min, amplitude $1.5 \mathrm{~cm})$. The experiment started when the shaker was placed into the climate chamber. Chamber temperature was maintained at $40{ }^{\circ} \mathrm{C}$ until the experiment was terminated.

\section{Source of worms}

Worms used in the experiment came from 14 naturally infected sticklebacks caught in ponds in Bochum, Germany. The resident fish population has been naturally exposed to the parasite for many years (M. Milinski, pers. comm.). Fish were maintained under long day conditions (16:8 h light:dark) in a 2701 flow through aquarium $(120 \times 45 \times 50 \mathrm{~cm})$ at a water temperature of $17^{\circ} \mathrm{C}$. Live Tubifex worms were provided ad libitum as food. Nine months after capture, fish were killed by a cerebrospinal cut, plerocercoids were removed, weighed (to the nearest $\mathrm{mg}$ ), and kept in culture medium (max. $3 \mathrm{~h}$ ) before transfer to the experimental set-up. Fish contained a total of 27 worms (one to six worms per fish). Four worms weighed less than $100 \mathrm{mg}$ and were not used in the experiment.

\section{Experimental set-up}

We used seven pairs and nine singles in the experiment (final sample size was eight singles because one died early during the experiment and was excluded from the analysis). Worms in pairs were matched for weight (average deviation from mean pair weight, $5.6 \% \pm 1.6 \mathrm{SE}$ ) and did not stem from the same donor fish. We balanced mean weights of the worms in the two treatment groups and their variances (paired worms: mean $\pm 1 \mathrm{SE}, 303 \pm 35 \mathrm{mg}, n=7$; single worms: $329 \pm 25 \mathrm{mg}, n=8 ; t=-0.62, d f=13, p>0.5)$. Similar numbers of worms in both treatment groups stem from singly, doubly and multiply infected sticklebacks $(3 \times 2$ Fisher exact test, $p=1.0)$. In contrast to the in vitro method, establishment rates in experimental final hosts are variable (56 to $100 \%$, Tierney and Crompton, 1992), suggesting that being in a multiple infection in the fish does not guarantee having a partner in the final host. It therefore appears unlikely that isolated worms from multiple infections are put in a situation to which they are not adapted.

\section{Data collection}

Upper and lower ends of the tubes were connected to silicon tubing. Every $6 \mathrm{~h}$ for the first 7 days and then daily until egg production had essentially ceased, we allowed $50 \mathrm{ml}$ of fresh culture medium to flow through the centrifuge tube replacing the used medium and effectively removing the eggs produced by the worms since the last rinse (rinse \#1 was done four instead of $6 \mathrm{~h}$ after the start of the experiment). 
Sampled eggs were placed in a refrigerator $\left(\right.$ at $4{ }^{\circ} \mathrm{C}$ ), and after settlement, eggs were rinsed with $0.9 \%$ sodium chloride solution, effectively leaving about $1 \%$ of the original medium in the tubes. Samples of rinses \#1 through \#8 were checked visually for the presence of eggs by filtering a third of the sample through a $0.45 \mu \mathrm{m}$ membrane filter. Eggs on the membrane were counted under a dissecting microscope. The first eggs started to appear in four tubes at rinse \#7 (i.e. $40 \mathrm{~h}$ after the start of the experiment) and in all but two tubes in rinse \#8 (46 h). Starting with rinse \#9 (52 h), we determined the number and size distribution of the eggs in the samples with an electronic particle analyser, a MultiSizer IIe (Coulter Electronics Limited, 1994), which gives a particle volume distribution with 256 size channels and the number of particles counted. We analyzed about $20 \%$ of the total sample. Egg volume is expected to range between 50,000 to $100,000 \mu^{3}$ (Wedekind et al., 1998) and we used a $280 \mu \mathrm{m}$ diameter orifice tube with the sampling range set between 30,700 and $131,600 \mu^{3}$. We analyzed an average of $1487(\mathrm{SE}=85)$ eggs per sample. The last three rinses (\#29 to \#31) were again analyzed by filtering and visually counting eggs as explained above.

\section{Data analysis}

To determine mean egg size per replicate, we calculated an overall particle size distribution by summing the particle distributions of rinses \#9 to \#28 (52 to $166 \mathrm{~h}$ ) for each tube. Bits of skin shed by the worms during and after metamorphosis can be within the measuring range, but are generally smaller in volume and much fewer than eggs. These particles may sometimes have caused a small left-hand skew in the particle size distributions. We compared the mean particle sizes of the whole distributions to the mean particle sizes excluding particles under a visually determined threshold. Particle sizes derived from both methods were highly correlated $\left(r^{2}=0.98\right.$, size difference $\left.<2 \%\right)$, and qualitative conclusions were not affected. We used the latter distributions to estimate mean egg size.

Analyses about the timing and magnitude of egg production were performed on the egg mass rather than egg number (in order to correct for the difference in egg size). Egg mass was calculated as egg number times the mean egg size per replicate. Because of the counting of the skin particles, egg mass may be overestimated by up to five percent (estimated from the number of particles under the threshold). However, the frequency of skin particles is expected to depend on the biomass in the tube and will not influence our results.

For each replicate we fitted a von Bertalanffy growth function (Ricklefs, 1967) to the square-root transformed cumulative egg mass production values (corrected for the number of worms in the replicate) using the nonlinear fitting platform of JMPIN 3.2.1. (Sall and Lehman, 1996). Transformation 
considerably improved the goodness of fit over untransformed values. Zero values before the start of the egg production were not included in the fit. The von Bertalanffy growth function, $y=a *(1-\exp (-k *(x-b)))$ consists of three parameters $(b, k$ and $a)$ that separately influence the shape and position of the curves (Fig. 1). Fitting this growth function to the collected data allowed us to estimate parameters $b, k$, and $a$ for each replicate of singles and pairs. Differences in each of these parameters between singles and pairs could have produced the difference in egg mass by day three observed by Wedekind et al. (1998). A difference in the starting point of reproduction would be indicated by a difference in parameter $b$ between the treatment groups, a lower rate of egg production in pairs would be indicated by a lower value in parameter $k$, and a lower magnitude of egg production in pairs would be indicated by a lower value of parameter $a$.

In order to assess whether the function fitted equally well in both treatment groups we compared (a) the $r^{2}$-values of the fits with a $t$-test, and (b) the residuals of the fits with a repeated measures ANOVA. In the ANOVA a treatment effect would indicate a difference in the overall goodness of fit between singles and pairs, and a time $\times$ treatment interaction would indicate that the goodness of fit differs at different locations of curves between the treatment groups. The same procedure revealed a lower goodness of fit when we fitted a logistic growth function (Ricklefs, 1967). The Gompertz function (Ricklefs, 1967) was about equivalent in goodness of fit, but had consistent problems in estimating parameter $b$.

We compared the estimated parameters with $t$-tests. All data are reported as means \pm 1 SE. We performed directed tests when we had clear predictions about the direction of effects from previous experiments (Rice and Gaines, 1994). Data were analyzed with JMPIN 3.2.1.

\section{Results}

In two tubes of each treatment group, the first eggs appeared after 34 to $40 \mathrm{~h}$ (i.e. at rinse \#7). Six hours later (rinse \#8) five and four tubes of the single and pair treatment groups respectively contained eggs. The last tubes first contained eggs (one of each treatment group) between 46 and $52 \mathrm{~h}$ (rinse \#9) On day 10 (rinse \#31) egg production had basically ceased and the experiment was stopped (Fig. 2A).

Egg size in pairs averaged higher than in singles (singles, $62,700 \pm 810 \mu \mathrm{m}^{3}$, pairs, $67,900 \pm 2,600 \mu \mathrm{m}^{3}$, Welch ANOVA $F_{1,7.5}, p=0.04$, directed). We also observed an initial difference in produced egg mass per worm until $70 \mathrm{~h}$ (singles, $3780 \pm 390 \times 10^{6} \mu \mathrm{m}^{3} /$ worm, pairs, $2440 \pm 380 \times 10^{6} \mu \mathrm{m}^{3} /$ worm, $t=2.4, d f=13, p=0.018$, directed), but this difference disappeared towards 


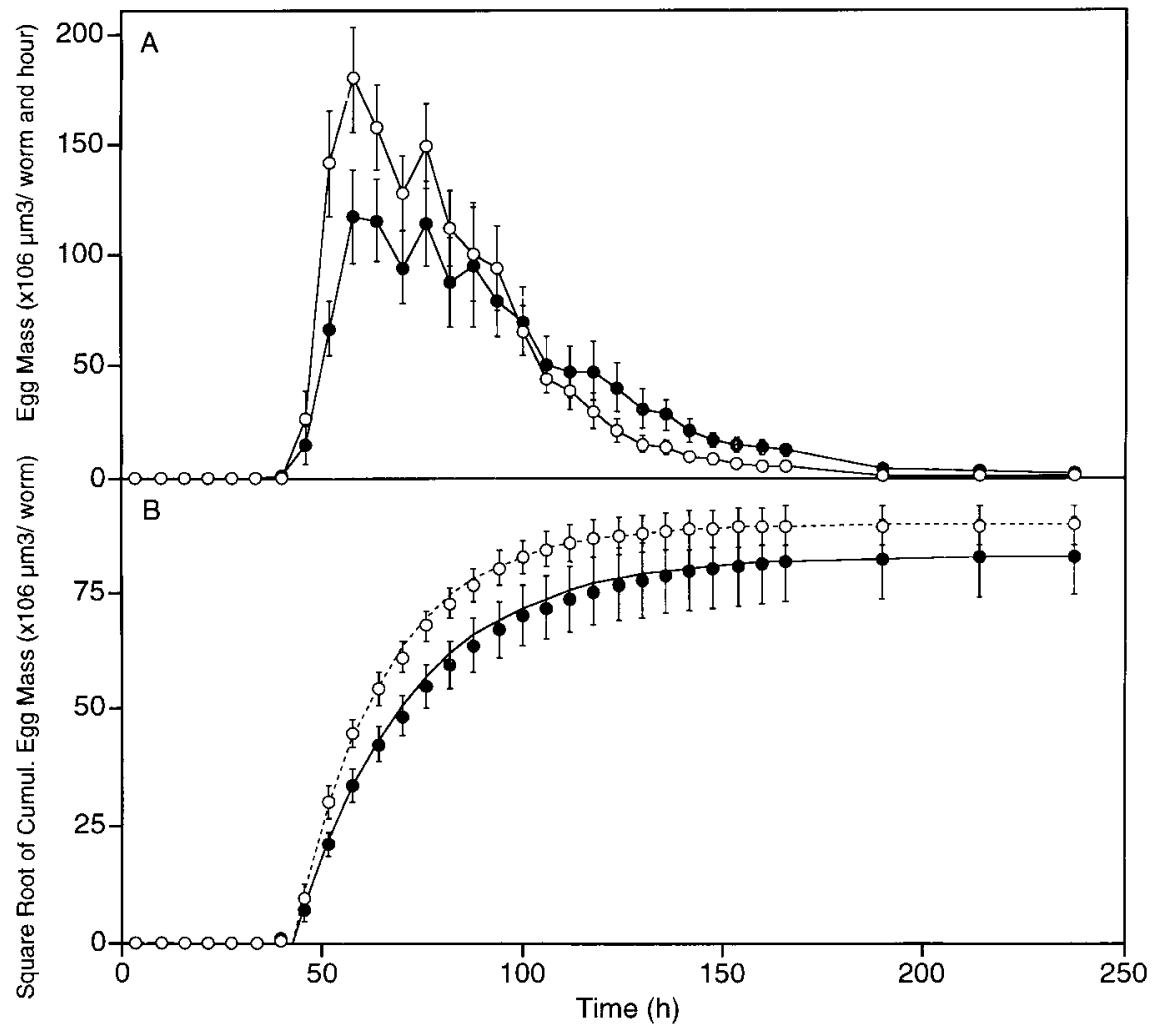

Figure 2. Observed dynamics and magnitude of lifetime egg production in S. solidus reproducing alone ( $n=8$, open circles) and in pairs ( $n=7$, closed circles). A: Egg mass production (egg number times mean egg size, mean $\pm 1 \mathrm{SE}$ ) per worm and hour. B: Cumulative egg mass production per worm (mean $\pm 1 \mathrm{SE}$ ). Superimposed are the von Bertalanffy growth functions using the mean parameters for singles (stippled line) and pairs (solid line).

the end of the experiment (singles, $8090 \pm 700$, pairs, $7230 \pm 1320, t=0.60$, $p>0.50$, two-tailed, statistical power using effect size of the comparison at $70 \mathrm{~h}$ is $62 \%$ ).

The von Bertalanffy growth function gave a good fit to the data (Fig. 2B). There was no clear indication for a difference in the goodness of fit between the treatment groups, as assessed by the mean $r^{2}$-values (singles, $0.9981 \pm 0.0003$, pairs, $0.9971 \pm 0.0010, t$-test on arcsine transformed values, $t=-1.06$, $d f=13, p>0.3$ ), or by the residuals of the fits (repeated measures ANOVA, between subjects: effect of treatment, $F_{1,13}=1.35, p>0.25$; within subjects, using Huynh-Feldt correction: treatment $\times$ time, $F_{3.9,51.1}=0.67, p>0.6$ ).

There was no significant difference in mean parameter $b$ between the two treatment groups (singles: $b=43.12 \pm 1.18$, pairs: $b=43.06 \pm 1.26$, $t=-0.04, d f=13, p>0.95$, Fig. 2B), indicating no difference in the starting 
point of reproduction. We, however, found a significantly higher mean parameter $k$ in singles than in pairs, indicating that the maximum egg production rate attained by singles was higher (singles: $k=0.0454 \pm 0.0025$, pairs: $k=0.0351 \pm 0.0030, t=-2.66, p<0.02$, Fig. 2B). The lower egg production rate in pairs did not appear to be related to the larger egg size they produce (ANCOVA: effect of treatment, $F_{1,12}=5.9, p<0.04$; effect of egg size, $\left.F_{1,12}=0.21, p>0.65\right)$. Neither did crowding appear to be responsible for this difference, since there was no significant negative correlation between the mean worm weights in the replicates and parameter $k$ (singles, $r_{\mathrm{s}}=0.31$, $p>0.45$, pairs, $r_{\mathrm{s}}=-0.50, p>0.25$ ).

There also was no significant difference in mean parameter $a$ between singles and pairs (singles: $a=89.4 \pm 4.2$, pairs: $a=82.6 \pm 8.9, t=-0.72, d f=13$, $p>0.45$, Fig. $2 \mathrm{~B}$ ), which is in accordance to the result derived directly from the data (above).

\section{Discussion}

The improved in vitro techniques we developed and used here allowed us to measure the timing of reproduction and the total lifetime egg output of worms in the two social situations with high temporal resolution. We were further able to replicate two key findings of an earlier study (Wedekind et al., 1998), namely that worms that were allowed to reproduce in pairs (a) produced larger eggs, and (b) produced less egg mass until three days after the start of the experiment. This again suggests a difference in the reproductive modes employed by isolated and paired worms. Together with the evidence we listed in the Introduction we feel confident that at least some outcrossing took place in the pairs. In the following we discuss the new findings of the current study, which allow us to distinguish between the different processes that may lead to point (b) above.

\section{Start of egg production}

Isolated individuals can be expected to have a relatively fixed reproductive strategy. In paired individuals, however, reproductive strategies may depend the mating partner (e.g. on its size, whether it will cross-fertilise, and how well it will co-operate in reproduction). Hence, there may be a need for mate assessment, mate choice and information transfer between the partners and this process may be time consuming and delaying the start of egg production. In our study, however, pairs and singles started egg production on average at about the same time.

For S. solidus waiting in the final host probably would be costly, because this species has no adhesive structures (hooks or suckers) and must constantly 
counteract the movements of the gut contents in order to stay in the host. Therefore, while waiting individuals would probably use energy they could otherwise allocate to reproduction. So both single and paired worms might be selected to start reproduction as early as possible. Alternatively, they may both wait for the same amount of time, but possibly due to different reasons.

\section{Rate of egg production}

Our study revealed a significantly lower egg production rate in paired individuals (Fig. 2), which can be interpreted in several ways. One form of mating system predicted for outcrossing simultaneous hermaphrodites is gamete trading (Leonard and Lukowiak, 1984), where parcels of gametes are reciprocally exchanged. This process may need time, causing lower egg production rates in pairs than singles. Another possible cause for the lower rate in pairs could be a difference in the mechanism of sperm transfer between the two social situations (i.e. a cost of cross-copulation). Some cestodes self-fertilise by copulating within the same segment whereas others self-fertilise between segments (Williams and McVicar, 1968). It is conceivable that the former mode speeds up reproduction in that the need for copulation is essentially avoided. As yet, it is not clear how self-fertilisation takes place in $S$. solidus. Another explanation for the lower rate is that larger eggs may take more time to produce. However, egg size did not seem to be related to the rate of egg production.

The points raised above suggest that the rate would be constrained in pairs, but it also appears possible that the reduced rate is an adaptive reaction to the different social situation. Pairs produce roughly twice as many eggs per unit of time as singles, which could lead to higher sib-competition between hatching larvae if eggs remain clumped after being excreted by the bird. A reduced egg production rate may be an adaptation to counteract that. This, however, would suggest that larger worms, which also produce more eggs per unit time, should have a lower egg production rate, which is not supported by our data.

Finally, there may be a possibility that the lower rate of egg production in pairs is caused by a crowding effect, for instance due to the accumulation of harmful metabolites or due to competition for nutrients. However, the culture medium functions as an effective buffer and we replaced the medium every $6 \mathrm{~h}$ thereby removing accumulated metabolites. Adult $S$. solidus have been suggested to take up glucose from culture medium (Hopkins, 1952), but at our rate of medium exchange worms would only have taken up an average of $3.5 \%$ (range 1.8 to $6.9 \%$ ) of the glucose present. Hence, competition for nutrients seems implausible in explaining the observed delay. Moreover, there was no significant negative correlation between the mean worm weights in the tubes and the rate of egg production in either treatment group, as would have been 
expected if crowding and/or competition for resources was causing the delay. Finally, the results of the current study are in very close agreement with the results of an earlier study (Wedekind et al., 1998), which experimentally controlled for possible influences of crowding by always placing two replicates (i.e. one single and one pair) per culture bottle. We therefore think that the observed delay in paired individuals is not caused by the higher biomass in the tubes of this treatment group.

\section{Magnitude of egg production}

In simultaneous hermaphrodites there is opportunity for conflicts of interest during reproduction (Michiels, 1998), and as mentioned earlier some helminths do partly self-fertilise, even if they have the opportunity to cross-fertilise (Nollen, 1975; Trouvé et al., 1996; Nollen, 1997). If so, the paired individuals could decide to increase allocation to the male function due to a risk of sperm competition. Such a phenotypically plastic response to the presence of a mating partner has recently been described for another parasitic helminth (Trouvé et al., 1999). The fact that the lifetime egg production was not significantly lower in pairs (both as estimated directly from the data and from the estimated parameter $a$, Fig. 2B) may indicate that paired worms co-operate during reproduction (i.e. that sperm competition is weak or absent). This is consistent with a recent study in which we found no significant change in male or female allocation in single vs. paired worms (L. Schärer, and C. Wedekind, unpubl. ms.). The same study found evidence for sperm competition in groups of three worms, but its occurrence in pairs remained unclear. Co-operation in reproduction could reduce sperm competition and could be achieved by gamete trading, which is in agreement with the observed difference in the rate of egg production mentioned above.

\section{Conclusions}

We found a lower rate of egg production in paired individuals of $S$. solidus compared to singles, which likely explains the initially lower egg mass production in pairs reported earlier (Wedekind et al., 1998). We suggest that the two most likely processes responsible for this observation are time costs associated with gamete trading in pairs and/or time costs caused by differences in the mode of sperm transfer between singles and pairs. We found no significant difference in the starting point of reproduction, nor did we find a significant difference in the final magnitude of egg mass production.

In vivo investigations with experimental final hosts have shown that worms can be passed out with the faeces after only $36 \mathrm{~h}$ and that egg output in faeces 
may cease early in the course of infection (Hopkins and Smyth, 1951; McCaig and Hopkins, 1963; Tierney and Crompton, 1992). Therefore pairs may sometimes not be able to catch up in egg production in the natural hosts (Fig. 2A). Early reproduction hence appears to be more valuable, and both singles and pairs should try to reproduce early. The fact that egg size in S. solidus decreases over time (Wedekind et al., 1998) also supports this notion. Our findings suggests a time cost associated with pairing in S. solidus, which in the case of gamete trading would represent a cost of sexual selection (Lloyd, 1980; Lewis, 1987). The absence of such a cost in singles may be able to partly compensate for possible adverse effects of self-fertilisation such as inbreeding depression. The cost in pairs could be partly compensated for by a higher fitness of outcrossed eggs, e.g. due to a higher hatching success in eggs stemming from pairs as opposed to singles (Wedekind et al., 1998) or due to increased genetic diversity among the offspring (Wedekind and Rüetschi, 2000). This suggests that the merits of self- vs. cross-fertilisation will depend not only on the often cited genetic consequences of the two reproductive modes, but also on behavioural patterns required to perform them.

\section{Acknowledgements}

We would like to thank Mira Christen, Peter Chylewski, Kati Turi Nagy and Nathalie Treichel for excellent lab assistance; Rolf Eggler, Julian Rauch and Peter Stettler for technical assistance and Michael Sänger of the Bundesamt für Gesundheit, Bern, for access to the MultiSizer. Martin Brinkhof, Annelis Lüscher and two anonymous reviewers provided useful comments on the manuscript. This project was supported by the Swiss National Science Foundation (Project No. 31-45733.95). CW thanks the "Berner Hochschulstiftung" and the Cloëtta Foundation for tide-over grants.

\section{References}

Andersson, M. and Iwasa, Y. (1996) Sexual selection. Trends Ecol. Evol. 11, 53-58.

Charlesworth, D. and Charlesworth, B. (1987) Inbreeding depression and its evolutionary consequences. Ann. Rev. Ecol. Syst. 18, 237-268.

Charnov, E.L. (1979) Simultaneous hermaphroditism and sexual selection. Proc. Natl. Acad. Sci. USA 76, 2480-2484.

Charnov, E.L. (1982) The Theory of Sex Allocation. Princeton University Press, Princeton, NJ, USA.

Coulter Electronics Limited. (1994) Coulter MultiSizer II, Reference Manual 9912433-C. Coulter Electronics Limited, Luton, England.

Cunningham, E.J.A. and Birkhead, T.R. (1998) Sex roles and sexual selection. Anim. Behav. 56, $1311-1321$. 
Ghiselin, M.T. (1969) The evolution of hermaphroditism among animals. Q. Rev. Biol. 44, 189208.

Greeff, J.M. and Michiels, N.K. (1999a) Low potential for sexual selection in simultaneously hermaphroditic animals. Proc. R. Soc. Lond. B 266, 1671-1676.

Greeff, J.M. and Michiels, N.K. (1999b) Sperm digestion and reciprocal sperm transfer can drive hermaphrodite sex allocation to equality. Am. Nat. 153, 421-430.

Hopkins, C.A. (1952) Studies on cestode metabolism. II. The utilization of glycogen by Schistocephalus solidus in vitro. Exp. Parasitol. 1, 196-213.

Hopkins, C.A. and Smyth, J.D. (1951) Notes on the morphology and life history of Schistocephalus solidus (Cestoda: Diphyllobothriidae). Parasitology 41, 283-291.

Jarne, P. and Charlesworth, D. (1993) The evolution of the selfing rate in functionally hermaphrodite plants and animals. Ann. Rev. Ecol. Syst. 24, 441-466.

Jarne, P. and Delay, B. (1990) Inbreeding depression and self-fertilisation in Lymnaea peregra (Gastropoda: Pulmonata). Heredity 64, 169-175.

Leonard, J.L. and Lukowiak, K. (1984) Male-female conflict in a simultaneous hermaphrodite resolved by sperm trading. Am. Nat. 124, 282-286.

Lewis, W.M.J. (1987) The cost of sex. In S.C. Stearns (ed) The evolution of sex and its consequences. Birkhäuser Verlag, Basel, Switzerland, pp. 33-57.

Lloyd, D.G. (1980) Benefits and handicaps of sexual reproduction. Evol. Biol. 13, 69-107.

McCaig, M.L.O. and Hopkins, C.A. (1963) Studies on Schistocephalus solidus. II. Establishment and longevity in the definitive host. Exp. Parasitol. 13, 273-283.

Michiels, N.K. (1998) Mating conflicts and sperm competition in simultaneous hermaphrodites. In T.R. Birkhead and A.P. Møller (eds) Sperm competition and sexual selection. Academic Press, London, England, pp. 219-254.

Nollen, P.M. (1975) Studies on the reproductive system of Hymenolepis diminuta using autoradiography and transplantation. J. Parasitol. 61, 100-104.

Nollen, P.M. (1983) Patterns of sexual reproduction among parasitic platyhelminths. Parasitology 86, $99-120$

Nollen, P.M. (1997) Mating behaviour of Echinostoma caproni and E. trivolvis in concurrent infections in hamsters. Int. J. Parasitol. 27, 71-75.

Rice, W.R. and Gaines, S.D. (1994) 'Heads I win, tails you lose': testing directional alternative hypotheses in ecological and evolutionary research. Trends Ecol. Evol. 9, 235-237.

Ricklefs, R.E. (1967) A graphical method of fitting equations to growth curves. Ecology 48, 978-983.

Sall, J. and Lehman, A. (1996) JMP Start Statistics: A Guide to Statistical and Data Analysis using $J M P$ and JMPIN Software. Duxbury Press, Belmont, USA.

Schmitt, J. and Ehrhardt, D.W. (1990) Enhancement of inbreeding depression by dominance and suppression in Impatiens capensis. Evolution 44, 269-278.

Smyth, J.D. (1946) Studies on tapeworm physiology. I. The cultivation of Schistocephalus solidus in vitro. J. Exp. Biol. 23, 47-73.

Smyth, J.D. (1952) Studies on tapeworm physiology. VI. Effect of temperature on the maturation in vitro of Schistocephalus solidus. J. Exp. Biol. 29, 304-309.

Smyth, J.D. (1954) Studies on tapeworm physiology. VII. Fertilization of Schistocephalus solidus in vitro. Exp. Parasitol. 3, 64-71.

Tierney, J.F. and Crompton, D.W.T. (1992) Infectivity of plerocercoids of Schistocephalus solidus (Cestoda: Ligulidae) and fecundity of the adults in an experimental definitive host, Gallus gallus. J. Parasitol. 78, 1049-1054.

Tomlinson, J. (1966) The advantage of hermaphroditism and parthenogenesis. J. Theor. Biol. 11, $54-58$.

Trouvé, S., Jourdane, J., Renaud, F., Durand, P. and Morand, S. (1999) Adaptive sex allocation in a simultaneous hermaphrodite. Evolution 53, 1599-1604.

Trouvé, S., Renaud, F., Durand, P. and Jourdane, J. (1996) Selfing and outcrossing in a parasitic hermaphrodite helminth (Trematoda, Echinostomatidae). Heredity 77, 1-8. 
Wedekind, C. (1997) The infectivity, growth, and virulence of the cestode Schistocephalus solidus in its first intermediate host, the copepod Macrocyclops albidus. Parasitology 115, 317-324.

Wedekind, C. and Rüetschi, A. (2000) Parasite heterogeneity affects infection success and the occurrence of within-host competition: an experimental study with a cestode (preprint edition). Evol. Ecol. Res. <http://www.evolutionary-ecology.com/forthcoming.html>

Wedekind, C., Strahm, D. and Schärer, L. (1998) Evidence for strategic egg production in a hermaphroditic cestode. Parasitology 117, 373-382.

Williams, H.H. and McVicar, A. (1968) Sperm transfer in tetraphyllidea (Platyhelminthes: Cestoda). Nytt Mag. Zool. 16, 61-71. 